\title{
Effect of early experience on neuronal and behavioral responses to con- and heterospecific odors in three closely related Mus taxa: epigenetic contribution in formation of precopulatory isolation
}

\author{
E. Kotenkova ${ }^{1 *}$, A. Maltsev ${ }^{1}$, A. Ambaryan ${ }^{1}$, A. Romachenko ${ }^{2}$ \\ ${ }^{1}$ Severtsov Institute of Ecology and Evolution RAS, Moscow, Russia \\ ${ }^{2}$ Institute of Cytology and Genetics SB RAS, Novosibirsk, Russia \\ *e-mail:evkotenkova@yandex.ru
}

Key words: Mus musculus, M. spicilegus, cross-fostering, early olfactory experience, main olfactory bulb, accessory olfactory bulb

Motivation and Aim: The individual learned phenotypic traits of parents and/or siblings, such as olfactory ones, result in the learner being able to discriminate its own species and sex of conspecifics. Olfactory plasticity to main social olfactory cues is limited to a critical period when exposure to odor might change responses to conand heterospecific odors. The olfactory system thus provides an attractive model to investigate processes involving interplay between genetic and epigenetic influences and their role in evolutionary process, especially development of precopulatory reproductive isolation. We alter maternal environment by cross-fostering. The objective was to evaluate the influence of early olfactory experience on the neuronal and behavioral response of males to con- and heterospecific odors of receptive females in two species M. musculus (subspecies musculus, wagneri) and M. spicilegus and thus, to determine the potential role of epigenetic contribution in formation of precopulatory isolation.

Methods and Algorithms: Males were reciprocally cross-fostered shortly after the birth and were tested for response to con- and heterospecific urine odors of estrous females using two-choice tests at 70-85 days of age. Neuronal activity of non- and cross-fostered males were evaluated at 90-110 days of age in main (MOB) and accessory olfactory bulbs (AOB) to con- and heterospecific female odor using fMRI (MEMRI).

Results: Non-fostered males of three taxa demonstrated a strong preference for odor of conspecific females. Male spicilegus raised by female musculus spent significantly more time investigating odor sources of heterospecific females. Wagneri-nursed spicilegus did not demonstrate significant choice of con - or heterospecific female odor. Non-fostered male spicilegus investigated longer odor of conspecific females in comparison with wagneri-nursed males. Wagneri-nursed spicilegus investigated more time urine odor of female wagneri in comparison with non-fostered male spicilegus. The level of MRI signal obtained from the evaluation of manganese accumulation in AOB neurons was significantly higher when the odor of urine of conspecific estrus females was exposed compared to urine exposure of heterospecific females. The response pattern changed to the opposite in males raised by heterospecific females. The maternal environment, including odor, had a greater effect on the level of MRI signal in the AOB than the genetic relationships of the recipient and the donor of the odor stimulus.

Conclusion: Behavioral and neuronal responses to con- and heterospecific odors changed in three closely related taxa of Mus as result of early experience. We demonstrated importance of early leaning in mate choice in adulthood in mice and possibility of epigenetic contribution in formation of precopulatory isolation.

Acknowledgements: Supported by the Russian Science Foundation grant No. 16-14-10269. 